\title{
La importancia de la Teoría de la Mente en la comprensión oral y comprensión lectora en estudiantes bilingües emergentes
}

\author{
The Importance of Theory of Mind in Oral and \\ Reading Comprehension in Emergent Bilingual \\ Students
}

\section{Ana Taboada Barber, Francisca Vizcaya-Jofré y Susan Lutz Klauda}

Universidad de Maryland, College Park, MD, Estados Unidos

\section{Resumen}

La Teoría de la Mente (ToM, por sus siglas en inglés) como aspecto relevante en el desarrollo socio-cognitivo ha sido ampliamente estudiada. Algunos resultados han sugerido una ventaja de los niños bilingües en el desarrollo de ToM respecto a sus pares monolingües. No obstante, la investigación sobre su rol predictivo en el desempeño en comprensión oral y lectora en estudiantes de primaria es menor. Este estudio evaluó una muestra de 84 niños bilingües emergentes, hispanohablantes, de tercer a quinto grado para identificar el rol predictivo de ToM en su comprensión oral y lectora en inglés, su segunda lengua, controlando por grado, vocabulario e identificación de palabras. ToM fue medida con una prueba de evaluación de intenciones, creencias y perspectivas de otros y con reconocimiento de emociones mediante expresiones faciales. Se emplearon pruebas estandarizadas de comprensión oral y lectora de pasajes. Los análisis de correlaciones bivariadas y regresión lineal jerárquica entre ToM y cada uno de los tipos de comprensión indicaron una predicción de ToM en ambos, controlando por grado, identificación de palabras y vocabulario. Esto destaca el rol de ToM en la oralidad y lectura de estudiantes bilingües emergentes y enfatiza su importancia para la enseńanza de la lectura en esta población.

Palabras clave: Teoría de la Mente (ToM); comprensión lectora; comprensión oral; estudiantes bilingües

Correspondencia a:

Ana Taboada Barber ataboada@umd.edu

University of Maryland, Department of Counseling, Special Education, and Higher Education, 1311 Benjamin Building, College Park, MD 20742.

(C) 2021 PEL, http://www.pensamientoeducativo.org - http://www.pel.cl 


\title{
Abstract
}

\begin{abstract}
Theory of Mind (ToM), as a relevant aspect in children's socio-cognitive development, has been widely studied. Some results have suggested that bilingual children have an advantage over their monolingual peers in development of ToM. However, there is less research regarding the predictive role of ToM in performance of oral and reading comprehension in elementary school students. This study evaluated a sample of 84 emergent bilingual, Spanish-speaking, third to fifth graders to identify the predictive role of ToM in their oral comprehension and reading comprehension in English, their second language, controlling by grade, vocabulary, and word identification. ToM was assessed using a test of other people's intentions, beliefs, and perspectives along with recognition of emotions through facial expressions. For comprehension, standardized measures of oral and reading comprehension were used. The bivariate correlations and hierarchical linear regression analyses between ToM and each of the types of comprehension indicated a prediction of ToM in both types of comprehension when controlling statistically for grade, word identification, and vocabulary. This highlights the role of ToM in oral language and reading variables in emergent bilingual students and emphasizes its importance for teaching of reading for this population.
\end{abstract}

Keywords: theory of mind (ToM); reading comprehension; oral comprehension; bilingual students

La Teoría de la Mente (ToM, por sus siglas en inglés) refiere a la capacidad de predecir y explicar la conducta de otras personas a partir de sus estados mentales, incluyendo creencias y deseos (Fletcher et al., 1995). Es una habilidad sociocognitiva que posibilita la interpretación del comportamiento de otras personas y la comunicación de los propios pensamientos, emociones y deseos a otros (Astington \& Edward, 2010, en Kim, 2020b). En términos conceptuales y operativos, se han distinguido distintos componentes de ToM que surgen a lo largo de las diferentes etapas del desarrollo evolutivo. La comprensión de falsas creencias de primer orden es una manifestación inicial de ToM, alrededor de los cuatro ańos (Wellman, Cross \& Watson, 2001), que muestra la capacidad de un niño para reconocer que distintas personas pueden tener creencias diferentes sobre la misma realidad y que estas creencias están sujetas a cambios (Astington, Pelletier \& Homer, 2002).

La comprensión de falsas creencias de segundo orden, manifestada entre los 5 y 7 años (Perner \& Wimmer, 1985; Sullivan, Zaitchik \& Tager-Flusberg, 1994), hace referencia al desarrollo de la conciencia sobre el hecho de que las personas poseen creencias sobre las creencias que otros tienen sobre el mundo y que estas, a su vez, pueden ser diferentes a las propias (Astington et al., 2002). Por último, en un nivel sociocognitivo más sofisticado, ToM avanzada, desarrollada entre los 8 y 10 años, corresponde a un conjunto de habilidades que denotan comprensión de falsas creencias de un orden más elevado, comprensión social, reconocimiento de estados emocionales y mentales y habilidades vinculadas con la toma de perspectiva (Osterhaus, Koerber \& Sodian, 2016). ToM es un componente sociocognitivo que se desarrolla desde las edades preescolares hasta los grados primarios tardíos.

Las primeras investigaciones sobre ToM se centraron en estudios de niños dentro del espectro autista (BaronCohen, 1989, 2000; Baron-Cohen, Leslie \& Frith, 1985; Leslie, 1987; Leslie \& Frith, 1988; Wulff, 1985) y fueron seguidas por investigaciones con niños neurotípicos, enmarcadas principalmente en la etapa inicial de su desarrollo (Miller, 2012), especialmente en las edades preescolares de 3 a 5 años. El estudio de ToM en edades de escuela primaria es más limitado (Hughes \& Devine, 2015) y la investigación empírica de ToM en poblaciones bilingües con respecto a su lectura es aún menor (Weimer et al., 2021). De hecho, es aún más escasa la investigación que desagregue subcomponentes o tipos de lectura (por ejemplo, literal versus inferencial o narrativa 
versus expositiva) en su relación con ToM en monolingües (Weimer et al., 2021) y, a nuestro saber, inexistente en bilingües. Dado que las poblaciones de estudiantes bilingües hispanohablantes en Estados Unidos tienen dificultades de comprensión lectora, en parte relacionadas con dificultades asociadas con la comprensión oral, la exploración de ToM con estas variables es de interés por su posible rol empírico, así como por sus implicancias para la enseñanza de la lectura en el aula.

Otros estudios muestran relaciones positivas, en su mayoría, entre ToM y funciones ejecutivas, tales como memoria de trabajo, flexibilidad de atención y control inhibitorio en edades preescolares (Carlson, Moses \& Breton, 2002; Hughes, 1998; Pérez-Zapata, Cuadra-Martínez, Valenzuela-Barrios \& Salgado-García, 2019) y escolares (Lecce, Bianco, Devine \& Hughes, 2017).

La relación de ToM con indicadores académicos en poblaciones de estudiantes de escuela primaria ha sido variada, incluyendo su vínculo con diversas variables sociocognitivas, como las circunstancias sociales de los niños en el aula, el desempeño (calificaciones), las estrategias académicas y la motivación ante el aprendizaje (Wellman, 2016). Pese a las relaciones entre ToM y los indicadores académicos, el desarrollo sociocognitivo de habilidades como ToM pocas veces se ve integrado en la escuela (Smogorzewska, Szumski \& Grygiel, 2020) y en relación con la lectura (por ejemplo, Kelly \& Taboada Barber, 2021). Específicamente, es relativamente limitada la cantidad de investigaciones sobre la relación de ToM con indicadores de lenguaje oral y de comprensión lectora simultáneamente, ambos indicadores académicos en escuelas primarias, medias y secundarias. Por ejemplo, entre 11 estudios en las últimas dos décadas sobre ToM y procesos de comprensión, solo cinco consideraron la relación de ToM con la comprensión lectora y la comprensión oral dentro del mismo estudio empírico (Atkison, Slade, Powell \& Levy, 2017; Ebert, 2020; Guajardo \& Cartwright, 2016; Kim, 2020a; Pelletier, 2006). Los estudios restantes consideraron solo un tipo de comprensión: oral o lectora.

La consideración de la relación empírica entre ToM y ambos tipos de comprensión, oral y lectora, es importante por dos motivos: primero, junto con la identificación de palabras, la comprensión oral en estadios tempranos del desarrollo del lenguaje es un predictor significativo de la comprensión lectora en edades posteriores en la escuela primaria (Cain, 2007; Kieffer, Biancarosa \& Mancilla Martínez, 2013; Language and Reading Research Consortium (LARRC), 2015; LARRC \& Chiu, 2018). Asimismo, existe evidencia contundente de que la influencia de la comprensión oral en la comprensión lectora se incrementa una vez que la identificación de palabras de los lectores está más desarrollada (García \& Cain, 2014; Vellutino, Tunmer, Jaccard \& Chen, 2007). Segundo, la comprensión oral -o comprensión lingüística-, uno de los dos componentes, junto con la decodificación, en el establecido modelo de Simple View of Reading (SVR) refiere a la habilidad de extraer y construir significados literales e inferenciales del discurso oral (Hoover \& Tunmer, 2020). En este artículo, adoptamos esta definición de comprensión oral o comprensión lingüística, como se refiere en la SVR, y optamos por el primer término. La comprensión oral no es solo un predecesor de la comprensión lectora, sino que, según el modelo SVR, es uno de los factores que, combinado con la decodificación, contribuye directamente a la comprensión lectora. Considerando el rol crucial de la comprensión oral en la comprensión lectora y, a su vez, la importancia de esta última para gran parte de los aprendizajes académicos, en este artículo proponemos considerar la relación de ToM, como una variable sociocognitiva, respecto de la comprensión oral y la comprensión lectora, respectivamente.

Este estudio se enfoca en estudiantes bilingües emergentes, lo que significa que son estudiantes que están convirtiéndose en bilingües en el proceso de ir aprendiendo el idioma social de su entorno y, a la vez, continuar desarrollando y comunicándose en su lengua nativa (García, Kleifgen \& Falchi, 2008). En otras palabras, manejan el inglés como medio de enseñanza en la escuela, pero hablan otra lengua en sus hogares; en este caso, español. Nos focalizamos en esta población de estudiantes bilingües hispanohablantes de escuela primaria por dos razones: en primer lugar, el manejo de dos o más lenguas y culturas ha llevado a una constatación empírica de que los niños bilingües tienden a tener una ToM más desarrollada que sus pares monolingües (Schroeder, 2018). 
Esta ventaja en ToM en niños bilingües emergentes se encontró aun cuando su nivel de dominio de inglés, su segunda lengua, fue controlada estadísticamente, destacando así el rol significativo de ToM. En segundo lugar, tenemos un interés particular en los procesos de comprensión de esta población y la escasez de estudios sobre el rol de ToM en esta justifica que se considere una exploración empírica. En las siguientes secciones consideramos la literatura previa sobre las relaciones de ToM con la comprensión oral, primero, y comprensión oral y compresión lectora luego, para culminar con estudios que consideraron ToM en poblaciones bilingües.

\section{ToM y comprensión oral}

Aunque la literatura empírica es relativamente reciente, existe evidencia de la relación entre ToM y la comprensión de narraciones orales de ficción, tanto en niños hispanohablantes como angloparlantes. Específicamente, de 11 estudios de las últimas dos décadas que se focalizaron en ToM y en los dos tipos de compresión, cinco incluyeron ambos tipos de comprensión y solo tres se focalizaron exclusivamente en comprensión oral. Dentro de estos tres estudios empíricos, Pelletier y Beatty (2015) exploraron la relación entre el nivel de desarrollo de ToM, medida mediante tareas de falsa creencia de primer y segundo orden y el nivel de comprensión oral de narraciones, fábulas específicamente, en una muestra de niños canadienses de 4 y 5 años. Para evaluar la comprensión, crearon una tarea en que los niños debían escuchar una fábula y observar imágenes relacionadas, para luego responder oralmente preguntas de comprensión y evaluación de la moraleja de la narración. Los resultados mostraron que, cuando se controló por vocabulario general, existía una asociación entre el desarrollo de ToM de segundo orden y la comprensión oral de fábulas, lo que sugiere que ToM juega un rol relevante en la habilidad de evaluar las intenciones de los personajes de este tipo de narraciones. No obstante, el estudio no ofrece información sobre los efectos del desarrollo de ToM en la comprensión lectora autónoma.

De manera similar, Strasser y del Río (2014) estudiaron el rol de ToM en la predicción de la comprensión de narraciones en niños de jardín de infantes de 5 años. A diferencia del estudio anterior, en este la comprensión oral fue medida mediante dos evaluaciones: una estandarizada de rememoración de cuentos y una adaptada por las investigadoras. En esta, los nińos observaban un libro álbum en que la historia es relatada únicamente por las imágenes, sin palabras, y respondían oralmente a preguntas de comprensión sobre personajes, detalles y procesos inferenciales. ToM fue medida mediante tareas de falsa creencia de contenido, falsa creencia explícita, creencia-emoción, emoción real-aparente y falsa creencia de segundo orden. Asimismo, se examinó el rol de funciones ejecutivas, monitoreo de la comprensión, así como profundidad y amplitud de vocabulario. Los resultados mostraron que, si bien ToM no predijo significativamente la comprensión de narraciones, se encontraron correlaciones estadísticamente significativas entre ToM y vocabulario, monitoreo de la comprensión, comprensión oral, memoria de trabajo e inhibición. Debido a las altas correlaciones con vocabulario y la ausente predicción de ToM en la comprensión oral (memoria para historias), Strasser y del Río (2014) sugieren que posiblemente ToM es una variable comúnmente confundida con la habilidad lingüística y sostienen que las habilidades lingüísticas de inferencias generales y de inferencias mentales son difíciles de aislar en niños de edades tempranas, lo que obstaculiza la identificación del efecto específico de la comprensión de los estados mentales en la comprensión oral.

Por último, Tompkins, Blosser y Downing (2020) estudiaron longitudinalmente el rol de ToM respecto de las habilidades de comprensión de historias y encontraron una correlación significativa positiva moderada entre ToM y la comprensión oral de historias concurrentes y la comprensión en un punto posterior. Sin embargo, cuando la edad y desarrollo del lenguaje de los participantes fueron controlados estadísticamente, no se encontró asociación significativa entre ToM y la comprensión oral de historias. 


\section{ToM, comprensión oral y comprensión lectora}

ToM se ha visto relacionada con la comprensión lectora en niños monolingües, tanto hispanohablantes (Ramos \& Crespo, 2008) como angloparlantes (Atkinson, Slade, Powell \& Levy, 2017; Boerma, Mol \& Jolles, 2017; Ebert, 2020; Guajardo \& Cartwright, 2016; Kim, 2020a) y bilingües (Pelletier, 2006) en diversos estudios empíricos que varían en la complejidad de sus análisis estadísticos y, por consecuencia, en el rol que ToM cumple. A un nivel únicamente correlacional, Ramos y Crespo (2008) estudiaron la relación entre ToM y comprensión lectora en niños chilenos de 8 y 9 años utilizando una medición construida con base en historias clásicas de falsas creencias de segundo orden. Encontraron una baja correlación, aunque estadísticamente significativa, entre ambas variables. Los autores concluyeron que la capacidad de representar internamente los estados mentales e intenciones de otros sería una condición elemental para que los niños desarrollen comprensión de las narraciones. Sin embargo, el estudio no considera otras variables de control que fortalezcan estos hallazgos.

En estudios donde sí se consideran variables de control o relaciones entre variables latentes en modelos de Structural Equation Modeling (SEM), las asociaciones entre ToM y la comprensión lectora varían con respecto a la edad de los participantes y al tipo de variables utilizadas en los modelos. Por ejemplo, en su investigación longitudinal, Guajardo y Cartwright (2016) evaluaron el nivel de desarrollo de ToM de primer orden de niños angloparlantes entre 3 y 5 ańos y luego la comprensión lectora y ToM de segundo orden a los 6 y 9 años. Las mediciones de ToM consistieron en tareas de falsa creencia de primer y segundo orden, mientras que para comprensión lectora se utilizaron instrumentos estandarizados. Los resultados indicaron que ToM de primer orden no contribuyó directamente a la comprensión lectora de párrafos, pero sí a la comprensión de oraciones aisladas cuando la memoria de trabajo, la flexibilidad cognitiva, el vocabulario y la decodificación fueron controlados estadísticamente. Notablemente, ToM en edad preescolar contribuyó a la comprensión lectora en edades posteriores, lo que señala tanto el rol de ToM como el de las funciones ejecutivas en la predicción longitudinal de la comprensión lectora.

ToM también juega un rol importante en su relación con la comprensión lectora en investigaciones que han identificado una asociación entre estas mediante variables intermediarias. Dentro de este marco, Ebert (2020) condujo un estudio longitudinal con un grupo de niños de los 3 a los 13 años en Alemania. Realizó mediciones en cuatro momentos: a los 3 años evaluó memoria de trabajo, habilidades no verbales y habilidades lingüísticas; a los 5 años, habilidades lingüísticas y ToM de primer y segundo orden; a los 12 años, vocabulario y ToM avanzada, y a los 13 ańos, comprensión lectora y oral. Los resultados señalaron que, aunque ToM medida en etapas iniciales no tuvo efecto en la comprensión lectora a los 13 años, ToM avanzada, evaluada a los 12 ańos, tuvo un pequeño efecto indirecto mediante la comprensión oral. Estos hallazgos se alinean con los de Atkinson y sus colegas (2017), cuyo estudio longitudinal evaluó a niños angloparlantes a los 4 y a los 6 años. Encontraron que ToM medida a los 4 años predijo indirectamente la comprensión lectora mediante la habilidad lingüística medida a los 6 años.

Por último, el estudio de Kim (2020a) midió ToM, comprensión oral y comprensión lectora evaluadas a través de pruebas estandarizadas, monitoreo de la comprensión, vocabulario, conocimiento gramatical y funciones ejecutivas en estudiantes angloparlantes en segundo y cuarto grado. Los resultados de SEM indicaron que el efecto indirecto de ToM en la comprensión lectora aumentó desde segundo a cuarto grado. Específicamente, se demostró que las variables fundacionales del lenguaje (vocabulario, gramática) y las variables cognitivas (memoria de trabajo, atención) difieren en su relación con ToM de acuerdo al grado/edad. Kim (2020a) concluyó que la demanda de vocabulario por parte de ToM decrece en cuarto grado y, en consecuencia, las diferencias individuales en memoria de trabajo explican varianza en ToM más allá del vocabulario y otras variables. 


\section{ToM en niños bilingüies}

La investigación empírica que vincula ToM con comprensión oral y comprensión lectora en niños bilingües es escasa. Sin embargo, en la última década ha proliferado un cuerpo de investigación empírica focalizado en las ventajas que tienen niños bilingües en ToM respecto de sus pares monolingües. Específicamente, un metaanálisis de 16 estudios empíricos que incluyó 1283 nińos reveló una pequeña ventaja en ToM para niños bilingües respecto de sus pares monolingües (Cohen's $d=0,22, p=0,05$ ) (Schroeder, 2018). Este estudio señaló que los primeros se desempeñan mejor que los últimos en ToM en edades tempranas, lo que indica que ToM es maleable y que puede ser facilitada por un entorno bilingüe. Hay tres explicaciones para esta ventaja. Una está basada en la idea de que el bilingüismo mejora el desarrollo y fortalece las funciones ejecutivas (Bialystok, 2016; Carlson \& Meltzoff, 2008) y que, a su vez, las funciones ejecutivas predicen ToM (Devine \& Hughes, 2014), de modo que el supuesto mayor control de atención puede usarse para regular los propios estados mentales, vale decir, las creencias y el conocimiento. La segunda explicación está basada en la idea de que el bilingüismo mejora la conciencia metalingüística (Ben-Zeev, 1977; Bialystok, 1988) y que esta se encuentra vinculada al desarrollo de ToM (Doherty, 2000; Doherty \& Perner, 1998). El hecho de que los bilingües entiendan que hay dos maneras, una en cada lengua, de referirse al mismo concepto puede facilitar el comprender que dos personas puedan tener un estado mental diferente respecto del mismo hecho y, por ende, diferir del estado mental que uno tiene respecto del mismo evento. La tercera explicación es la sociopragmática, que sostiene que del mismo modo que los bilingües llegan a comprender en edades tempranas que dos personas pueden hablar ya sea una de las dos lenguas o ambas, transfieren esta noción a la idea de que dos personas pueden tener estados mentales diferentes o similares. Las tres explicaciones predicen una ventaja de los bilingües en ToM, que ha sido algunas veces avalada por estudios empíricos. En el metaanálisis mencionado, es notorio que cuando se controló estadísticamente por el nivel de proficiencia, la ventaja en ToM de los niños bilingües se vio incrementada respecto del análisis inicial (Cohen's $d=0,58, p=0,001$ ), lo que sugiere que ToM puede ser potenciada por la experiencia bilingüe.

\section{El estudio}

En este estudio nos focalizamos en la relación que ToM mantiene con la comprensión oral y la comprensión lectora en una muestra de nińos bilingües de tercer a quinto grado de primaria. Este estudio se enmarca dentro de la literatura anterior precisamente porque ToM puede resultar una habilidad a desarrollar en estudiantes bilingües de escuela primaria, dada su posible relación con la lectura y la oralidad. Este estudio busca entender la relación entre ToM y los dos tipos de comprensión de forma más acabada en esta población. Sin embargo, nuestro interés no busca demostrar la ventaja de ToM en niños bilingües en comparación con los monolingües, sino proveer datos empíricos que indiquen si ToM predice la comprensión oral y la comprensión lectora en niños bilingües emergentes. Lo anterior, dada la importancia de estas habilidades académicas para todos los estudiantes, pero particularmente para aquellos que tienen dificultades específicas con ellas, como sucede con la mayoría de los estudiantes bilingües hispanohablantes en Estados Unidos. De este modo, dos preguntas de investigación guiaron nuestro estudio:

1. ¿Puede ToM explicar varianza en la comprensión oral en inglés (segunda lengua) de estudiantes bilingües emergentes de escuela primaria cuando grado, vocabulario e identificación de palabras son controlados estadísticamente?

2. ¿Puede ToM explicar varianza en la comprensión lectora en inglés (segunda lengua) de estudiantes bilingües emergentes de escuela primaria cuando grado, vocabulario e identificación de palabras son controlados estadísticamente? 
Dada la naturaleza exploratoria del estudio, preliminar en el área, usamos vocabulario e identificación de palabras como variables de control por el rol prominente que tienen en ambos tipos de comprensión. Controlamos por grado, pues ToM puede variar entre las edades correspondientes a tercer (7-8 años) y quinto grado (10 años). Asimismo, elegimos explorar solo el nivel predictivo de ToM respecto de los dos tipos de comprensión, dejando para estudios posteriores su potencial rol mediador. Consideramos que el temprano estadio de investigación del tema en esta población justifica nuestro enfoque de regresión simple con cada constructo por separado.

\section{Método}

\section{Participantes}

Participaron 84 estudiantes hispanos bilingües emergentes, de habla hispana e inglesa, de tercer a quinto grado de una escuela primaria pública suburbana de alto nivel de vulnerabilidad en un estado de la costa atlántica de Estados Unidos. Según los registros escolares, más del 90\% de los estudiantes de la escuela eran hispanos y más del 90\% recibían comidas gratuitas, indicador de bajo nivel socioeconómico. La muestra se seleccionó aleatoriamente de los 113 estudiantes de habla hispana de los grados tercero a quinto de la escuela que participaban en un proyecto más amplio sobre el desarrollo de lectura de estudiantes bilingües. Todos reportaron hablar español en el hogar y sus padres eran hispanohablantes nativos. De acuerdo con los registros escolares, cuatro de los participantes presentaban discapacidad, pero pudieron participar en las evaluaciones del mismo modo que el resto de los estudiantes incluidos en la muestra. La tabla 1 muestra la información demográfica.

La enseñanza de la lectura en los cursos inferiores de primaria incluye trabajo fonético diario y de comprensión lectora tres días a la semana. Si bien en los grados superiores había un énfasis mayor en la comprensión lectora, las estrategias de comprensión fueron un foco de enseñanza en todos los cursos. Se utilizaba el sistema de niveles de lectura A-Z de Fountas y Pinnell (2007) junto con lecciones de lectura guiada. Los estudiantes del programa de Inglés para Hablantes de Otros Idiomas (ESOL, por sus siglas en inglés) recibían instrucción de inglés oral fuera del aula mínimo tres veces por semana.

Tabla 1

Información demográfica de la muestra

Muestra completa $(\mathrm{n}=84)$

\begin{tabular}{lcc}
\hline \multicolumn{2}{c}{ Información demográfica } & $\%$ \\
\hline Grado & 3 & $38,1 \%$ \\
\hline 4 & $36,9 \%$ \\
\hline Género & 5 & $25,0 \%$ \\
\hline & Femenino & \\
\hline & Masculino & $47,6 \%$ \\
\hline & No reportado & $48,8 \%$ \\
\hline
\end{tabular}




\begin{tabular}{cc}
\hline Estatus en ESOL & \\
\hline Activo & $73,8 \%$ \\
\hline Egr. $<2$ a & $6 \%$ \\
\hline Egr. $>2$ a & $10,7 \%$ \\
\hline Evaluado & $6 \%$ \\
\hline No reportado & $3,6 \%$ \\
\hline Idioma hablado $\quad$ & $19,0 \%$ \\
\hline Español e inglés & $56,0 \%$ \\
\hline Mayormente español & $10,7 \%$ \\
\hline Mayormente inglés & $11,9 \%$ \\
\hline Solo español & $2,4 \%$ \\
\hline No reportado & \\
\hline Idioma de lectura $\quad 2,1 \%$ \\
\hline Español e inglés & $2,4 \%$ \\
\hline Mayormente español & $16,7 \%$ \\
\hline Mayormente inglés & $2,4 \%$ \\
\hline Solo español & $69 \%$ \\
\hline Solo inglés & $2,4 \%$ \\
\hline
\end{tabular}

Nota: Los porcentajes pueden no sumar 100 debido a redondeo. Estatus en ESOL: Activo = actualmente en el programa ESOL; Egr. $<2 \mathrm{a}=$ egresado dentro de los últimos 2 años académicos. Egr. $>2$ a = egresado hace más de 2 años académicos. Evaluado = Evaluado para el programa ESOL y considerado no elegible. Idioma hablado = idioma(s) que la familia del estudiante habla en casa. Idioma de lectura = idioma(s) en que el estudiante lee en casa.

\section{Procedimientos y mediciones}

\section{Identificación de palabras}

Se utilizó la subprueba de identificación de letras y palabras de las Pruebas de Aprovechamiento de la batería Woodcock Johnson-IV (WJ-IV) (Schrank, Mather \& McGrew, 2014). Esta requiere que los estudiantes lean de manera individual una lista de letras y palabras en inglés presentadas por escrito. Se asigna un punto por cada respuesta correcta, con un puntaje total de 78 . La confiabilidad de la prueba original medida según el método de dos mitades corresponde a un rango de 0,94 a 0,98 para estudiantes de 6 a 10 años (McGrew, LaForte $\&$ Schrank, 2014).

\section{Vocabulario}

Se utilizó la subprueba de vocabulario sobre dibujos de la batería WJ-IV (Schrank et al., 2014). Los estudiantes deben nombrar las imágenes correspondientes a objetos en 54 ítems. La dificultad de la tarea aumenta durante la prueba, en la medida en que las imágenes se vuelven menos familiares. Se asigna un punto por cada respuesta correcta, con un puntaje total de 54 . La confiabilidad de la prueba original medida según el método de dos mitades corresponde a un rango de 0,77 a 0,78 para estudiantes de entre 6 a 10 años (McGrew et al., 2014). La consistencia interna para esta muestra fue de 0,79 . 


\section{Comprensión oral}

Para medir la comprensión oral en inglés se utilizó el subtest homónimo de WJ-IV (Schrank et al., 2014) y para medirla en español, el subtest homónimo de Pruebas de Aprovechamiento de la batería Woodcock Muñoz (WMLS-R) (Schrank et al., 2005). En estas pruebas, los estudiantes escuchan pasajes breves y proveen una palabra faltante basándose en pistas sintácticas y semánticas. El puntaje es el número total de respuestas correctas de 33 ítems. Los coeficientes de confiabilidad según el método de dos mitades para 6 a 10 años se encuentran en el rango de 0,78 a 0,83 para la prueba en inglés (McGrew et al., 2014) y 0,92 a 0,97 para la prueba en español (Schrank et al., 2005). En la muestra actual, la consistencia interna (Alfa de Cronbach) fue de 0,77 para la prueba en inglés y de 0,88 para la prueba en español.

\section{Comprensión lectora}

Se utilizó la subprueba de comprensión de pasajes de la batería WJ-IV (Schrank et al., 2014) en inglés. Los ítems aumentan su complejidad en la medida en que avanza la prueba y son de tres tipos: asociar un ícono con una imagen real, identificar la imagen que representa un enunciado compuesto por una a tres palabras y proveer la palabra faltante tras leer silenciosamente pasajes cortos incompletos. Se asigna un punto por respuesta correcta, con 52 como puntaje máximo. La confiabilidad de la prueba original medida según el método de dos mitades corresponde a un rango de 0,89 a 0,98 para nińos de 6 a 10 años (McGrew et al., 2014). En esta muestra, la consistencia interna fue de 0,93 .

\section{Teoría de la Mente (ToM)}

Se utilizó el subtest de NEPSY-II (Korkman, Kirk \& Kemp, 2007a), que mide el desempeño asociado con la habilidad de entender las intenciones, creencias y perspectivas de otros y, a su vez, el reconocimiento de emociones a través de expresiones faciales. La subescala verbal contiene 15 ítems que incluyen escuchar situaciones, 11 de ellas con apoyo pictórico, y responder preguntas que implican conocimiento del punto de vista de otro. La subescala contextual considera seis ítems que consisten en observar imágenes que muestran un contexto social y seleccionar la foto que representa la emoción de la persona de la imagen cuyo rostro no se encuentra visible. Esta prueba tiene una confiabilidad de test-retest de 0,77 para niños de primaria (Korkman, Kirk \& Kemp, 2007b). Cada ítem vale 1 a 2 puntos y el puntaje corresponde al número total de puntos obtenidos. En este estudio, dos de los ítems fueron excluidos debido a un mal funcionamiento psicométrico, resultando en un puntaje máximo de 24 puntos. La confiabilidad (Alfa de Cronbach) para la escala final es de 0,66.

\section{Procedimiento}

Los estudiantes fueron evaluados por un equipo especializado durante la segunda mitad del año escolar. Todas las evaluaciones se realizaron en una habitación con bajo nivel de ruido en las escuelas durante 75 a 90 minutos. El primer día se completaron los instrumentos de lenguaje y lectura y el segundo día, la evaluación de ToM. Las instrucciones fueron dadas en inglés, dado que es el idioma de la escuela y del entorno, a excepción de la prueba de comprensión oral en español. Todos los participantes tenían suficiente nivel de dominio del idioma para tomar parte en las tareas administradas. El equipo de evaluadores se entrenó durante cuatro sesiones en los procedimientos de administración. Previo a la recolección de datos, se obtuvo aprobación del Institutional Review Board y el consentimiento de las escuelas, apoderados y profesores. 


\section{Resultados}

\section{Estadística descriptiva}

En la tabla 2 se observa la estadística descriptiva y las correlaciones bivariadas para todas las variables utilizadas en los análisis principales y para comprensión oral en español. ToM mostró correlaciones significativas leves a moderadas con todas las otras variables. Se evidencia que ToM correlacionó significativamente con comprensión oral en español, $r(82)=0,24, p=0,03$, aunque no tan fuertemente como con comprensión oral en inglés, $r(82)$ $=0,46, p \leq 0,001$. Así, el análisis principal se enfocó en la comprensión oral en inglés.

Tabla 2

Matriz de correlación y estadistica descriptiva

\begin{tabular}{cccccccc} 
& ToM & CL & CO-I & IP & V & CO-E & Gr \\
\hline ToM & - & & & & & \\
\hline CL &, $49^{* * *}$ & - & & & & \\
\hline CO-I &, $46^{* * *}$ &, $51^{* * *}$ & - & & & \\
\hline IP &, $36^{* * *}$ &, $82^{* * *}$ &, $36^{* * *}$ & - & & & \\
\hline $\mathrm{V}$ &, $36^{* * *}$ &, $52^{* * *}$ &, $74^{* * *}$ &, $41^{* * *}$ & - & & \\
\hline $\mathrm{CO}-\mathrm{E}$ &, $24^{*}$ &, $25^{*}$ &, $25^{*}$ &, $24^{*}$ &, $22^{*}$ & - & \\
\hline $\mathrm{Gr}$ &, $34^{* *}$ &, $29^{* *}$ &, $34^{* *}$ &, $32^{* *}$ &, $33^{* *}$ &, $32^{* *}$ & - \\
\hline$P$ & 17,66 & 24,93 & 14,96 & 46,70 & 24,29 & 13,01 & 3,87 \\
\hline$D E$ & 3,39 & 4,51 & 3,51 & 8,81 & 3,90 & 5,51 &, 79 \\
\hline
\end{tabular}

Nota $:$ ToM = Teoría de la Mente; CL = comprensión lectora inglés; CO-I = comprensión oral inglés; $\mathrm{IP}$ = identificación de palabras; $\mathrm{V}=$ vocabulario; $\mathrm{CO}-\mathrm{E}=$ comprensión oral español; $\mathrm{Gr}=\mathrm{Grado}$.

${ }^{*} p \leq, 05 ;{ }^{* *} p \leq, 01 ;{ }^{* * *} p \leq, 001$.

\section{Relación entre ToM y comprensión oral}

Para abordar nuestras dos preguntas de investigación, empleamos una regresión lineal jerárquica. Para la primera pregunta de investigación, con comprensión oral como variable dependiente, ingresamos las variables independientes en tres pasos. El paso 1 consistió en grado, el paso 2 incorporó identificación de palabras y vocabulario, y el paso 3, las anteriores más ToM (tabla 3). Este análisis mostró que ToM predijo significativamente comprensión oral, tras controlar por grado, identificación de palabras y vocabulario, $\beta=$ $0,21, p=0,01$ (ver tabla 3, modelo 3). Vocabulario también fue un predictor significativo en los modelos 2 y 3 , mientras que el grado únicamente fue significativo cuando fue ingresado por sí solo en el modelo 1 . La identificación de palabras no fue un predictor significativo en estos análisis. ToM agregó 3\% a la varianza predicha en comprensión oral más allá de las tres variables de control, con los cuatro predictores en conjunto explicando 59\% de la varianza en comprensión oral. 
Tabla 3

Predicción de comprensión oral en inglés

\begin{tabular}{ccccccccccc} 
& & \multicolumn{9}{c}{$\beta$} \\
\cline { 3 - 5 } Modelo & $\begin{array}{c}\text { Variable } \\
\text { independiente }\end{array}$ & $\mathrm{Gr}$ & $\mathrm{IP}$ & $\mathrm{V}$ & $\mathrm{ToM}$ & $\mathrm{R}^{2}$ & $\Delta \mathrm{R}^{2}$ & $\mathrm{dfs}$ & $\Delta \mathrm{F}$ \\
\hline 1 & $\mathrm{Gr}$ &, $34^{* *}$ & & & &, 11 &, 11 & 1,82 & $10,60^{* *}$ \\
\hline 2 & $\mathrm{Gr}+\mathrm{IP}+\mathrm{V}$ &, 10 &, 05 &, $69^{* * *}$ & &, 56 &, 44 & 2,80 & $39,81^{* * *}$ \\
\hline 3 & $\mathrm{Gr}+\mathrm{IP}+\mathrm{V}+\mathrm{ToM}$ &, 05 &, 01 &, $64^{* * *}$ &, $21^{*}$ &, 59 &, 03 & 1,79 & $6,52^{*}$ \\
\hline
\end{tabular}

Nota: $\mathrm{Gr}=$ grado; IP = identificación de palabras; $\mathrm{V}=$ vocabulario; ToM = Teoría de la Mente.

${ }^{*} p \leq, 05 ;{ }^{* *} p \leq, 01 ;{ }^{* * *} p \leq, 001$.

\section{Relación entre ToM y comprensión lectora}

El análisis de regresión para la segunda pregunta de investigación fue construido de manera similar, pero con comprensión lectora como variable dependiente (tabla 4). ToM predijo significativamente la comprensión lectora, controlando por grado, identificación de palabras y vocabulario, $\beta=0,19, p=0,004$ (ver tabla 4, modelo 3). La identificación de palabras y el vocabulario también fueron predictores significativos en los modelos 2 y 3 , mientras que el grado fue significativo únicamente cuando fue ingresado por sí solo en el modelo 1. ToM agregó $3 \%$ a la varianza predicha en comprensión lectora más allá de las tres variables de control, con los cuatro predictores en conjunto explicando $75 \%$ de la varianza en comprensión lectora.

Tabla 4

Predicción de comprensión lectora en inglés

\begin{tabular}{|c|c|c|c|c|c|c|c|c|c|}
\hline \multirow[b]{2}{*}{ Modelo } & \multirow[b]{2}{*}{$\begin{array}{c}\text { Variable } \\
\text { independiente }\end{array}$} & \multicolumn{4}{|c|}{$\beta$} & \multirow[b]{2}{*}{$\mathrm{R}^{2}$} & \multirow[b]{2}{*}{$\Delta \mathrm{R}^{2}$} & \multirow[b]{2}{*}{$\mathrm{dfs}$} & \multirow[b]{2}{*}{$\Delta \mathrm{F}$} \\
\hline & & Gr & IP & $\mathrm{V}$ & ToM & & & & \\
\hline 1 & Gr &, $29^{* *}$ & & & & ,08 & ,08 & 1,82 & $7,47^{* *}$ \\
\hline 2 & $\mathrm{Gr}+\mathrm{IP}+\mathrm{V}$ &,- 02 &, $74^{* * *}$ &, $23^{* * *}$ & &, 72 & ,64 & 2,80 & $90,36^{* * *}$ \\
\hline 3 & $\mathrm{Gr}+\mathrm{IP}+\mathrm{V}+\mathrm{ToM}$ &,- 06 &, $70^{* * *}$ &, $19^{* *}$ &, $19^{* *}$ &, 75 &, 03 & 1,79 & $8,77^{* *}$ \\
\hline
\end{tabular}

Nota: $\mathrm{Gr}=$ grado; $\mathrm{IP}=$ identificación de palabras; $\mathrm{V}$ = vocabulario; ToM = Teoría de la Mente.

${ }^{*} p \leq, 05 ;{ }^{* *} p \leq, 01 ;{ }^{* * *} p \leq, 001$. 


\section{Discusión}

En este estudio se exploró la contribución de ToM a la comprensión oral y a la comprensión lectora con dos instrumentos estandarizados en estudiantes bilingües emergentes hispanohablantes de cursos de escuela primaria en Estados Unidos. La consideración de esta población sitúa al estudio como uno de los primeros en establecer la relación entre ToM y variables de comprensión en bilingües emergentes. Los resultados de este estudio mostraron que, así como vocabulario, ToM fue un predictor significativo de comprensión oral en inglés, al explicar un $3 \%$ de su varianza tras controlar por grado, vocabulario e identificación de palabras. El modelo en su conjunto explicó un $59 \%$ de la varianza en comprensión oral. De manera similar, se encontró que, así como el vocabulario y la identificación de palabras, ToM fue un predictor significativo de comprensión lectora en inglés, al explicar un $3 \%$ de su varianza tras controlar por las mismas variables. El modelo en su conjunto explicó un $75 \%$ de la varianza en comprensión lectora. Por un lado, estos hallazgos son relevantes en un marco amplio por el rol que las habilidades sociocognitivas como ToM juegan en la educación y en el desarrollo de la lectura. Estudiarlas en poblaciones bilingües, en donde han sido identificadas como una ventaja por sobre poblaciones monolingües (Schroeder, 2018) permite considerar el rol de ToM en el fortalecimiento del desempeńo académico y psicosocial de los lectores en formación. Específicamente, los estudiantes bilingües hispanohablantes tienden a tener dificultades relacionadas con la comprensión lectora, por lo que la posibilidad de utilizar su ventaja en habilidades como ToM para fortalecer su desempeño en lectura requiere ser explorada. Por otro lado, estos resultados deben ser considerados dentro de un marco limitado, dado que la porción de varianza exclusivamente explicada por ToM fue solo de 3\% en ambos constructos, dejando lugar para múltiples interpretaciones respecto de estas relaciones.

\section{Predicción de comprensión oral}

Los resultados de este estudio apoyan y coinciden con los de Pelletier y Beatty (2015) en el sentido de que ToM contribuyó a la comprensión oral más allá del vocabulario y de un indicador de la capacidad de lectura (identificación de palabras), con el vocabulario contribuyendo significativamente, pero no así la identificación de palabras. El estudio actual también extiende estos hallazgos centrados en niños de 4 y 5 años a estudiantes mayores en escuela primaria. Además, Pelletier y Beatty (2015) emplearon una muestra en la que $60 \%$ de los niños hablaban una variedad de primeros idiomas además del inglés, pero pocos eran hispanohablantes, por lo que este estudio aporta evidencia respecto del rol que ToM tiene en la comprensión oral de hispanohablantes que aprenden inglés como lenguaje de su entorno, distinto al idioma del hogar.

En particular, nuestro estudio difiere de Pelletier y Beatty (2015) en los tipos de mediciones empleadas, lo cual puede considerarse en el limitado monto de varianza explicado por ToM en nuestros hallazgos. Específicamente, usamos la identificación de palabras como variable de control, mientras que Pelletier y Beatty (2015) emplearon un instrumento que evalúa los conceptos de pre-alfabetización, el conocimiento del alfabeto y la comprensión de significados básicos. Además, en este estudio utilizamos para nuestra medición de comprensión oral una medida más amplia que la usada por Pelletier y Beatty (2015), quienes se centraron en la comprensión de narraciones, específicamente fábulas. Asimismo, consideramos medidas de ToM más generales que no se encasillan en tareas de creencia de primer o segundo orden, sino que contemplan habilidades asociadas con la capacidad de entender las intenciones, creencias y perspectivas de otros y con la interpretación de emociones a través de expresiones faciales. En cambio, Pelletier y Beatty (2015) utilizaron medidas separadas de primer y segundo orden de ToM y encontraron que solo ToM de segundo orden era un predictor único significativo. Así y todo, es posible que nuestra medición de ToM explique la varianza limitada (3\%), si bien estadísticamente significativa, en los dos constructos de interés: comprensión oral y comprensión lectora. Es posible que si nuestros instrumentos de comprensión hubieran estado más alineados con la medición de ToM usada por estos autores, o si esta hubiera contemplado un componente inferencial marcado respecto de comportamientos en situaciones sociales que 
requieren leer intenciones o leer expresiones faciales, la predicción de varianza explicada por ToM en estos constructos hubiera sido mayor. Por ejemplo, en la historia Querido Juno de Soyung Pak (2001), un nińo llamado Juno se comunica con su abuela en Corea mediante una serie de dibujos, mientras que ella utiliza cartas, fotos y juguetes para responderle para que él entienda el significado. En este tipo de historias se refuerzan temas de comunicación, diversidad lingüística y cultural y habilidades relacionales, todos componentes importantes en ToM como constructo sociocognitivo. Una medición de comprensión que considerara estas dimensiones hubiera, posiblemente, incrementado la correlación y a su vez la predicción de ToM, dado que las habilidades específicas compartidas por el predictor y el constructo predicho habrían estado más alineadas.

Junto con lo anterior, los resultados actuales contrastan con los de Strasser y del Río (2014), quienes encontraron que en niños preescolares chilenos ToM no contribuyó a la comprensión de historias. Las autoras, sin embargo, emplearon varios predictores además de los estudiados aquí, incluyendo tres medidas de funciones ejecutivas, así como monitoreo de comprensión, inferencia y amplitud y profundidad de vocabulario, y dejando fuera mediciones de lectura. No obstante, encontraron correlaciones positivas pero débiles en cuanto a significancia entre ToM y sus mediciones de comprensión de historias ( 0,18 y 0,21, respectivamente). El contraste con nuestros resultados puede estar dado por la inclusión en el estudio de Strasser y del Río (2014) de tres funciones ejecutivas y habilidades de comprensión (como monitoreo de la comprensión y capacidad inferencial), todas habilidades cognitivas que son, o bien componentes claves del lenguaje oral y escrito (por ejemplo, Cain \& Oakhill, 2007) o fuertes predictores de la comprensión oral y la comprensión lectora tanto en estudiantes monolingües como estudiantes bilingües desde primer hasta cuarto grado (véase, Taboada Barber, Klauda \& Stapleton, 2020a; Taboada Barber et al., 2020b). Esta configuración de predictores deja un rol mínimo para el rol predictivo de variables sociocognitivas y más distantes de la comprensión como ToM, como es factible haya sido el caso en el estudio de Strasser y del Río (2014).

\section{Predicción de comprensión lectora}

Este estudio extiende el trabajo previo que encontró correlación positiva entre ToM y la comprensión lectora en estudiantes hispanohablantes de primaria en Chile (Ramos \& Crespo, 2008) al controlar por factores no considerados anteriormente, como la identificación de palabras y el vocabulario, variables clave contribuyentes a la comprensión lectora, así como el grado. Además, los resultados actuales extienden anteriores conducidos con estudiantes monolingües angloparlantes de escuela primaria (Atkison et al., 2017; Guajardo \& Cartwright, 2016), ya que indican que, en el caso de hispanohablantes que son bilingües emergentes, las relaciones predictivas entre ToM y comprensión lectora existen en su segunda lengua (inglés), aun cuando se controla por fuertes predictores de la lectura, como la decodificación y el vocabulario en la segunda lengua. Al considerar la ventaja que estudios preliminares muestran respecto de ToM en estudiantes bilingües, se abre la posibilidad de explorar su posible rol como potenciador de otras habilidades vinculadas al desarrollo académico de esta población, posibilidad que utilizaría ToM como un activo y permitiría distanciarse del modelo de déficit de aprendizaje que se asocia frecuentemente con esta población.

Es informativo considerar los hallazgos actuales a la luz de otros estudios centrados en el rol de ToM junto con una serie de variables sociocognitivas y generales, como las funciones ejecutivas, en nińos europeos o europeoamericanos (Atkinson et al., 2017; Boerma et al., 2017; Ebert, 2020; Guajardo \& Cartwright, 2016; Kim, 2020a). Por ejemplo, el estudio de Guajardo y Cartwright (2016) señalado anteriormente examinó longitudinalmente el papel de ToM en la comprensión lectora en niños principalmente de preescolar y primaria monolingües en Estados Unidos. Encontraron que ToM como falsa creencia de segundo orden y comprensión lectora, medidas concurrentemente, correlacionaban positivamente $(r=0,55)$ en la infancia media. Sin embargo, ninguna de las variables de ToM contribuyó de forma exclusiva a la comprensión lectora cuando se incluyó en su análisis 
la función ejecutiva de flexibilidad cognitiva, lo que sugiere nuevamente un rol predominante de estas funciones cognitivas y reduce la posibilidad de contribución o explicación de varianza para ToM cuando las anteriores se incluyen en modelos de regresión. Esto fue confirmado por las autoras mediante un análisis que arrojó evidencia de que la flexibilidad cognitiva podría estar enmascarando la contribución de ToM a la comprensión lectora en la infancia media. En su análisis, ToM representó de manera única 14\% de la varianza en la comprensión lectora al incluir varios controles (edad, educación materna, vocabulario, decodificación) y excluir flexibilidad cognitiva.

Ebert (2020) también examinó el papel de ToM en múltiples puntos de tiempo en niños alemanes de 3 a 13 años y descubrió que, en modelos complejos y multivariados, ToM solo contribuyó indirectamente a la comprensión lectora mediante la comprensión oral cuando sus participantes eran adolescentes jóvenes. Es probable que si en este estudio hubiéramos incluido la comprensión oral como variable de control, la predicción de ToM en la comprensión lectora se hubiera visto altamente disminuida o hubiera desaparecido. Sin embargo, nuestro objetivo no era considerar el rol de ToM con comprensión oral como variable mediadora, sino simplemente observar la relación de ToM con cada uno de estos constructos por separado. Nuevamente, consideramos que dado el estado preliminar de la investigación de ToM en estudiantes bilingües emergentes, comenzar con un análisis de regresión que distinga las relaciones de ToM con cada constructo de interés es un buen primer paso. Sumamos a este razonamiento el hecho de que metodólogos en mediación sostienen que los análisis de variables mediadoras no deben ser una exploración empírica exclusivamente, sino que, así como los modelos de SEM se basan en un modelo conceptual o teórico previo, se hace necesario por lo menos una hipótesis o un marco teórico adecuado que fundamente el mecanismo de mediación entre tres variables (Curran \& Hancock, 2020). Así, dado que este es un nuevo set de relaciones entre variables en una población que no había sido estudiada anteriormente, optamos por un análisis de regresión que considere los constructos de interés independientemente antes que un modelo de mediación.

\section{Futuros estudios}

Dado que esta investigación presenta un nuevo dominio en el estudio de la lectura y educación de estudiantes bilingües, los pasos futuros contemplan varias alternativas. Una de estas es la medición de ToM entre las lenguas del entorno del hogar o primera lengua de los niños y sus resultados académicos. En este estudio no contamos con estas variables, lo que imposibilitó dicho análisis. Una segunda opción es la comparación del rol de ToM en niños bilingües emergentes y en aquellos completamente bilingües (bilingües balanceados), ya que su rol podría ser más prominente en los bilingües balanceados, considerando que tienden a tener más desarrollada la literacidad en ambos idiomas. Esta idea sería conmensurable con la de Kim (2020a), quien encontró que ToM fue un mayor contribuyente a la comprensión lectora en cuarto que en segundo grado, lo que sugiere que con un mayor desarrollo del lenguaje (en el caso de Kim debido a la edad y no por dominio del idioma), ToM puede llegar a ser más importante para la comprensión cuando tanto la primera como la segunda lengua están más equitativamente desarrolladas. Una tercera alternativa a considerar es el rol de las funciones ejecutivas y ToM en bilingües. Si bien no exploramos este aspecto en nuestro estudio, la evidencia de la ventaja en funciones ejecutivas que se observa en los bilingües sugiere que posiblemente esta sea una dimensión de interés dentro de la investigación lingüística. Los resultados de estudios anteriores indican que una constelación de funciones ejecutivas se ha de considerar para evitar una minimización de la posible contribución de ToM. Dado el incremento en estudios empíricos enfocados en las funciones ejecutivas y su rol en la comprensión lectora de bilingües emergentes (Taboada Barber et al., 2020a; Taboada Barber et al., 2020b; Kieffer, Mancilla-Martínez \& Logan, 2021), considerar su rol vis a vis ToM presentaría una serie de predicciones interesantes para lograr un conocimiento más acabado de la comprensión lectora en bilingües emergentes. 


\section{Conclusión}

La creciente diversidad lingüística de los contextos educativos presenta nuevos desafíos tanto a investigadores como a actores pedagógicos dentro de las escuelas para responder a las demandas específicas que los estudiantes presentan en su aprendizaje. En el ámbito de la literacidad, una diversidad de factores y constructos tienen efecto en el desarrollo de la comprensión oral y la comprensión lectora. Como señalan nuestros resultados, ToM es uno de ellos, por lo que es relevante identificar y diseñar estrategias didácticas que promuevan esta habilidad sociocognitiva, así como explorar a través de qué mecanismos se puede sacar provecho de la ventaja en ToM de los estudiantes bilingües emergentes, quienes están especialmente necesitados de un enfoque pedagógico que se distancie de un modelo de déficit. Junto con estas implicancias prácticas, los hallazgos también destacan la importancia de elaborar marcos pedagógicos para la integración de las diversas dimensiones del desarrollo e invitan a revisar en qué medida los instrumentos curriculares a disposición del profesorado incorporan las habilidades sociocognitivas como parte del desarrollo lingüístico de los estudiantes.

Financiamiento: La investigación fue apoyada por el Instituto de Ciencias de la Educación del Departamento de Educación de Estados Unidos, a través del Fondo R305A160280 para la Universidad de Maryland. Las opiniones expresadas corresponden a los autores y no representan la visión del Instituto o del Departamento de Educación de Estados Unidos.

El artículo original fue recibido el 23 de enero de 2021

El artículo revisado fue recibido el 30 de junio de 2021

El artículo fue aceptado el 9 de julio de 2021

\section{Referencias}

Astington, J. W., Pelletier, J. \& Homer, B. (2002). Theory of mind and epistemological development: The relation between children's second-order false-belief understanding and their ability to reason about evidence. New Ideas in Psychology, 20(2-3), 131-144. https://doi.org/10.1016/S0732-118X(02)00005-3

Atkinson, L., Slade, L., Powell, D. \& Levy, J. P. (2017). Theory of mind in emerging reading comprehension: a longitudinal study of early indirect and direct effects. Journal of Experimental Child Psychology, 164, 225-238. https://doi.org/10.1016/j.jecp.2017.04.007

Baron-Cohen, S. (1989). Are autistic children "behaviorists"? An examination of their mental-physical and appearancereality distinctions. Journal of Autism and Developmental Disorders, 19(4), 579-600. https://doi.org/10.1007/BF02212859

Baron-Cohen, S. (2000). Theory of mind and autism: A fifteen year review. En S. Baron-Cohen, H. Tager-Flusberg \& D. J. Cohen (Eds.). Understanding other minds: Perspectives from developmental cognitive neuroscience (2a ed.) (pp. 3-20). Oxford, Inglaterra: Oxford University Press.

Baron-Cohen, S., Leslie, A. M. \& Frith, U. (1985). Does the autistic child have a "theory of mind"? Cognition, 21(1), 37-46. https://doi.org/10.1016/0010-0277(85)90022-8

Ben-Zeev, S. (1977). The influence of bilingualism on cognitive strategy and cognitive development. Child Development, 48(3), 1009-1018. https://doi.org/10.2307/1128353

Bialystok, E. (1988). Levels of bilingualism and levels of linguistic awareness. Developmental Psychology, 24(4), 560-567. https://doi.org/10.1037/0012-1649.24.4.560 
Bialystok, E. (2016). The signal and the noise: Finding the pattern in human behavior. Linguistic Approaches to Bilingualism, 6(5), 517-534. https://doi.org/10.1075/lab.15040.bia

Boerma, I. E., Mol, S. E. \& Jolles, J. (2017). The role of home literacy environment, mentalizing, expressive verbal ability, and print exposure in third and fourth graders' reading comprehension. Scientific Studies of Reading, 21(3), 179-193. https://doi.org/10.1080/10888438.2016.1277727

Cain, K. (2007). Deriving word meanings from context: Does explanation facilitate contextual analysis? Journal of Research in Reading, 30(4), 347-359. https://doi.org/10.1111/j.1467-9817.2007.00336.x

Cain, K. \& Oakhill, J. (2007). Children's comprehension problems in oral and written language: A cognitive perspective. Nueva York, NY: Guilford Press.

Carlson, S. M. \& Meltzoff, A. N. (2008). Bilingual experience and executive functioning in young children. Developmental Science, 11(2), 282-298. https://doi.org/10.1111/j.1467-7687.2008.00675.x

Carlson, S. M., Moses, L. J. \& Breton, C. (2002). How specific is the relation between executive function and theory of mind? Contributions of inhibitory control and working memory. Infant and Child Development: An International Journal of Research and Practice, 11(2), 73-92. https://doi.org/10.1002/icd.298

Curran, P. \& Hancock, G. (12 de octubre de 2020). Mediation and the art of squid spleening (Temporada 2, episodio 8) [Episodio de audio podcast]. En Quantitude. Recuperado de https:/quantitudepod.org/listen/

Devine, R. T. \& Hughes, C. (2014). Relations between false belief understanding and executive function in early childhood: A meta-analysis. Child Development, 85(5), 1777-1794. https://doi.org/10.1111/cdev.12237

Doherty, M. (2000). Children's understanding of homonymy: Metalinguistic awareness and false belief. Journal of Child Language, 27(2), 367-392. https://doi.org/10.1017/S0305000900004153

Doherty, M. \& Perner, J. (1998). Metalinguistic awareness and theory of mind: Just two words for the same thing? Cognitive Development, 13(3), 279-305. https://doi.org/10.1016/S0885-2014(98)90012-0

Ebert, S. (2020). Theory of mind, language, and reading: Developmental relations from early childhood to early adolescence. Journal of Experimental Child Psychology, 191, 104739. https://doi.org/10.1016/j.jecp.2019.104739

Fletcher, P. C., Happe, F., Frith, U., Baker, S. C., Dolan, R. J., Frackowiak, R. S. \& Frith, C. D. (1995). Other minds in the brain: a functional imaging study of "theory of mind" in story comprehension. Cognition, 57(2), 109-128. https://doi.org/10.1016/0010-0277(95)00692-R

Fountas, I. C. \& Pinnell, G. S. (2007). Fountas and Pinnell Benchmark Assessment System 2. Portsmouth, NH: Heinemann.

García, J. R. \& Cain, K. (2014). Decoding and reading comprehension: A meta-analysis to identify which reader and assessment characteristics influence the strength of the relationship in English. Review of Educational Research, 84(1), 74-111. https://doi.org/10.3102/0034654313499616

García, O., Kleifgen, J.A. \& Falchi, L. (2008). From English language learners to emergent bilinguals. Equity Matters: Research Review No.1. Nueva York, NY: Campaign for Educational Equity, Teachers College, Columbia University.

Guajardo, N. R. \& Cartwright, K. B. (2016). The contribution of theory of mind, counterfactual reasoning, and executive function to pre-readers' language comprehension and later reading awareness and comprehension in elementary school.Journalof ExperimentalChild Psychology, 144,27-45. https://doi.org/10.1016/j.jecp.2015.11.004

Hoover, W. A. \& Tunmer, W. E. (2020). The cognitive foundations of reading and its acquisition: A framework with applications connecting teaching and learning. Dordrecht, Holanda: Springer.

Hughes, C. (1998). Executive function in preschoolers: Links with theory of mind and verbal ability. British Journal of Developmental Psychology, 16(2), 233-253. https://doi.org/10.1111/j.2044-835X.1998.tb00921.x

Hughes, C. \& Devine, R. T. (2015). Individual differences in theory of mind from preschool to adolescence: Achievements and directions. Child Development Perspectives, 9(3), 149-153. https://doi.org/10.1111/cdep.12124

Kelly, J. H. \& Taboada-Barber, A. (2021). Interactive read alouds with narrative text: Teaching inference-making with Theory of Mind. TEACHING Exceptional Children. https://doi.org/10.1177/0040059920987876

Kieffer, M.J., Biancarosa, G. \& Mancilla-Martínez, J. (2013). Roles of morphological awareness in English reading comprehension for Spanish speaking language minority learners: Exploring partial mediation by vocabulary and reading fluency. Applied Psycholinguistics, 34(4), 697-725. https://doi.org/10.1017/S0142716411000920 
Kieffer, M. J., Mancilla-Martinez, J. \& Logan, J. K. (2021). Executive functions and English reading comprehension growth in Spanish-English bilingual adolescents. Journal of Applied Developmental Psychology, 73, 101238. https://doi.org/10.1016/j.appdev.2021.101238

Kim, Y. -S. G. (2020a). Hierarchical and dynamic relations of language and cognitive skills to reading comprehension: Testing the direct and indirect effects model of reading (DIER). Journal of Educational Psychology, 112(4), 667-684. https://doi.org/10.1037/edu0000407

Kim, Y. -S. G. (2020b). Theory of mind mediates the relations of language and domain-general cognitions to discourse comprehension.Journalof ExperimentalChild Psychology, 194,104813.https://doi.org/10.1016/j.jecp.2020.104813

Korkman, M., Kirk, U. \& Kemp, S. (2007a). NEPSY-II: A developmental neuropsychological assessment. San Antonio, TX: Harcourt Assessment, The Psychological Corporation.

Korkman, M., Kirk, U. \& Kemp, S. (2007b). NEPSY-II: Clinical and interpretive manual. San Antonio, TX: Harcourt Assessment, The Psychological Corporation.

Language and Reading Research Consortium (LARRC). (2015). Learning to read: Should we keep things simple? Reading Research Quarterly, 50(2), 151-69. https://doi.org/10.1002/rrq.99

Language and Reading Research Consortium (LARRC) \& Chiu, Y.D. (2018). The simple view of reading across development: Prediction of grade 3 reading comprehension from prekindergarten skills. Remedial and Special Education, 39(5), 289-303. https://doi.org/10.1177/0741932518762055

Lecce, S., Bianco, F., Devine, R. T. \& Hughes, C. (2017). Relations between theory of mind and executive function in middle childhood: A short-term longitudinal study. Journal of Experimental Child Psychology, 163, 69-86. https://doi.org/10.1016/j.jecp.2017.06.011

Leslie, A. M. (1987). Pretense and representation: The origins of "theory of mind". Psychological Review, 94(4), 412-426. https://doi.org/10.1037/0033-295X.94.4.412

Leslie, A. M. \& Frith, U. (1988). Autistic children's understanding of seeing, knowing and believing. British Journal of Developmental Psychology, 6(4), 315-324. https://doi.org/10.1111/j.2044-835X.1988.tb01104.x

McGrew, K. S., LaForte, E. M. \& Schrank, F. A. (2014). Woodcock Johnson IV Technical Manual. Rolling Meadows, IL: Riverside.

Miller, S. A. (2012). Theory of mind: Beyond the preschool years. Nueva York, NY: Psychology Press.

Osterhaus, C., Koerber, S. \& Sodian, B. (2016). Scaling of advanced theory-of-mind tasks. Child Development, 87(6), 1971-1991. https://doi.org/10.1111/cdev.12566

Pak, S. (2001). Dear Juno. Nueva York, NY: Penguin Group.

Pelletier, J. (2006). Relations among theory of mind, metacognitive language, reading skills and story comprehension in L1 and L2 learners. En A. Antonietti, O. L. Sempio, \& A. Marchetti (Eds.) Theory of mind and language in developmental contexts (pp. 77-92). Boston, MA: Springer.

Pelletier, J. \& Beatty, R. (2015). Children's understanding of Aesop's fables: relations to reading comprehension and theory of mind. Frontiers in Psychology, 6, 1448. https://doi.org/10.3389/fpsyg.2015.01448

Pérez-Zapata, D. I., Cuadra-Martínez, D. J., Valenzuela-Barrios, C. \& Salgado-García, P. (2019). Teoría de la mente y su relación con inhibición conductual en pre-escolares chilenos. Estudios de Psicología, 40(2), 453-463. https://doi.org/10.1080/02109395.2019.1589084

Perner, J. \& Wimmer, H. (1985). “John thinks that Mary thinks that ..." attribution of second-order beliefs by 5 - to 10 -yearoldchildren.Journalofexperimentedchildpsychology,39(3),437-471.https://doi.org/10.1016/0022-0965(85)90051-7

Ramos, C. \& Crespo, N. (2008). Niños que leen, niños que comprenden intenciones: relación entre la comprensión lectora y la teoría de la mente. Enunciación, 13(1), 53-60. https://doi.org/10.14483/22486798.1261

Schrank, F. A., Mather, N. \& McGrew, K. S. (2014). Woodcock-Johnson IV Tests of Achievement. Rolling Meadows, IL: Riverside.

Schrank, F. A., McGrew, K. S., Ruef, M. L., Alvarado, C. G., Muńoz-Sandoval, A. F. \& Woodcock, R. W. (2005). Overview and technical supplement (Batería III Woodcock-Muñoz Assessment Service Bulletin No. 1). Rolling Meadows, IL: Riverside Publishing.

Schroeder, S. R. (2018). Do bilinguals have an advantage in theory of mind? A meta-analysis. Frontiers in Communication, 3(36), 1-8. https://doi.org/10.3389/fcomm.2018.00036 
Smogorzewska, J., Szumski, G. \& Grygiel, P. (2020). Theory of mind goes to school: Does educational environment influence the development of theory of mind in middle childhood? Plos One, 15(8), e0237524. https://doi.org/10.1371/journal.pone.0237524

Strasser, K. \& del Río, F. (2014). The role of comprehension monitoring, theory of mind, and vocabulary depth in predicting story comprehension and recall of kindergarten children. Reading Research Quarterly, 49(2), 169-187. https://doi.org/10.1002/rrq.70

Sullivan, K., Zaitchik, D. \& Tager-Flusberg, H. (1994). Preschoolers can attribute second-order beliefs. Developmental psychology, 30, 395-402. https://doi.org/10.1037/0012-1649.30.3.395

Taboada Barber, A., Klauda, S. L. \& Stapleton, L. M. (2020a). Cognition, engagement, and motivation as factors in the reading comprehension of dual language learners and English speakers: Unified or distinctive models? Reading and Writing, 33, 2249 -2279. https://doi.org/10.1007/s11145-020-10034-4

Taboada Barber, A., Cartwright, K. B., Stapleton, L. M., Klauda, S. L., Archer, C. \& Smith, P. (2020b). Direct and indirect effects of executive functions, reading engagement, and higher order strategic processes in the reading comprehension of dual language learners and English monolinguals. Contemporary Educational Psychology, 61, 1-17. https://doi.org/10.1016/j.cedpsych.2020.101848

Tompkins, V., Blosser, M. K. \& Downing, M. (2020). False belief understanding and narrative comprehension in the preschool years. Cognitive Development, 56, 100936. https://doi.org/10.1016/j.cogdev.2020.100936

Vellutino, F. R., Tunmer, W. E., Jaccard, J. \& Chen, R. (2007). Components of reading ability: Multivariate evidence for a convergent skills model of reading development. Scientific Studies of Reading, 11(1), 3-32. https://doi.org/10.1080/10888 430709336632

Weimer, A. A., Warnell, K. R., Ettekal, I., Cartwright, K. B., Guajardo, N. R. \& Liew, J. (2021). Correlates and antecedents of theory of mind development during middle childhood and adolescence: An integrated model. Developmental Review, 59, 100945. https://doi.org/10.1016/j.dr.2020.100945

Wellman, H. M. (2016). Cognición social y educación: teoría de la mente. Pensamiento Educativo.Revista de Investigación Latinoamericana, 53(1), 1-23. https://doi.org/10.7764/PEL.53.1.2016.2

Wellman, H. M., Cross, D. \& Watson, J. (2001). Meta-analysis of theory of-mind development: The truth about false belief. Child development, 72, 655-684. https://doi.org/10.1111/1467-8624.00304

Wulff, S. B. (1985). The symbolic and object play of children with autism: A review. Journal of autism and developmental disorders, 15(2), 139-148. https://doi.org/10.1007/BF01531600 